\title{
Avaliação hemodinâmica intra-operatória na cirurgia de revascularização miocárdica sem auxílio da circulação extracorpórea
}

\author{
Ricardo Carvalho LIMA*, Mozart Augusto Soares de ESCOBAR* ${ }^{*}$ Renato Fábio DELLA SANTA*, \\ Roberto DINIZ* ${ }^{\star}$ Giusseppe D’ACONDA**, Jacob BERGSLAND**, Tomas SALERNO**
}

RBCCV 44205-503

Lima R C, Escobar M A S, Della Santa R S, Diniz R, D’Aconda G, Bergsland J, Salerno T - Avaliação hemodinâmica intra-operatória na cirurgia de revascularização miocárdica sem auxílio da circulação extracorpórea. Rev Bras Cir Cardiovasc 2000; 15 (3): 201-11.

RESUMO: Objetivo: Analisar o comportamento hemodinâmico do coração na revascularização do miocárdio sem a utilização da circulação extracorpórea, através da cateterização da artéria pulmonar com cateter de San-Ganz.

Material e Métodos: No período de agosto de 1991 a junho de 1999, foram operados 616 pacientes portadores de angina do peito, que foram submetidos a revascularização do miocárdio sem a utilização da circulação extracorpórea. Em 18 pacientes foram estudados os parâmetros hemodinâmicos intra-operatórios.

Resultados: A freqüência cardíaca manteve-se elevada durante todos os momentos de posicionamento do coração ( $p=0,0007)$. O débito cardíaco ao longo do procedimento apresentou variação mínima nos diversos momentos de posicionamento do coração e exposição das artérias coronárias. Entretanto, com o coração na posição normal final, observou-se um aumento importante do débito cardíaco $(p=0,010)$. A pressão arterial média apresentou-se diminuída em todos os momentos do procedimento de exposição das artérias coronárias $(\mathrm{p}=0,022)$. A pressão arterial pulmonar apresentou-se diminuída durante todos os momentos de mobilização (NS). A pressão capilar pulmonar oscilou bastante durante a exposição das coronárias (NS). A pressão venosa central comportou-se de maneira mais variada durante a exposição das artérias (NS). A resistência vascular sistêmica apresentou-se diminuída durante todo o procedimento $(p=0,0001)$. A resistência vascular pulmonar apresentou-se diminuída em todos os momentos do procedimento $(p=0,002)$. O "stroke volume" apresentou-se inalterado durante a anastomose da interventricular anterior e só se observaram diferenças estatísticas na coronária direita $(p=0,002)$ e artéria circunflexa $(p=0,0006)$ e seus ramos. $O$ índice cardíaco apresentou-se diminuído durante o procedimento $(p=0,0011)$.

CONCLUSÕES: A) A técnica presente permite a mobilização máxima do coração sem indução de instabilidade hemodinâmica. B) A melhora de alguns parâmetros de hemodinâmica ao final do procedimento pode ser justificada: 1) devido à resposta à revascularização miocárdica; 2) decorrente da liberação de catecolaminas após a manipulação do coração nas diversas posições; 3) decorrente da liberação de mediadores vasoativos depois da tração prolongada do pericárdio.

DESCRITORES: Revascularização miocárdica, métodos. Coração, fisiopatologia. Circulação extracorpórea. Hemodinâmica. Período intra-operatório.

Trabalho realizado no "General Buffalo Hospital", State University of New York, USA.

Apresentado ao 270 Congresso Nacional de Cirurgia Cardíaca. Rio de Janeiro, RJ, 23 a 25 de março de 2000.

Laureado com o "Prêmio Nacional de Cirurgia Cardíaca - 2000"

* Do UNITÓRAX - Real Hospital Português. Recife, PE, Brasil.

** Da University at Buffalo Hospital, State University of New York, USA.

Endereço para correspondência: Ricardo de Carvalho Lima. Rua dos Navegantes, 1515/101. Boa Viagem, Recife, PE, Brasil. CEP 51021-010. e.mail: rclima@elogica.com.br 
Lima R C, Escobar M A S, Della Santa R S, Diniz R, D’Aconda G, Bergsland J, Salerno T - Avaliação hemodinâmica intra-operatória na cirurgia de revascularização miocárdica sem auxílio da circulação extracorpórea. Rev Bras Cir Cardiovasc 2000; 15 (3): 201 -11.

\section{INTRODUÇÃO}

Trabalhos pioneiros de KOLESSOV (1), ANKENEY (2), TRAPP \& BISARYA (3), BUFFOLO et al. (4) e BENETTI (5) deram início a experiência com a revascularização miocárdica sem utilização da circulação extracorpórea. Em 1993, iniciou-se, no UNITÓRAX, trabalho cooperativo com o grupo do Instituto do Coração de Maceió, representando, assim, experiência pioneira no Nordeste do Brasil (6). Nessa experiência conjunta, ficou demonstrado que o procedimento preconizado por BUFFOLO et al. (4) e BENETTI (5) era reproduzível por outras equipes.

Desde que BENETTI (5) demonstrou pela primeira vez sua experiência, denominando-a de cirurgia coronária minimamente invasiva, um grande interesse em todo o mundo para realização desse procedimento. A operação consistia em dissecar a artéria torácica interna com auxílio do toracoscópio e realização da anastomose da artéria torácica interna na interventricular anterior, via uma pequena toracotomia esquerda. A cirurgia minimamente invasiva serviu para mostrar que a cirurgia de revascularização sem CEC, via esternotomia, é também um procedimento menos invasivo, uma vez que o elemento mais agressivo é a circulação extracorpórea.

Entretanto, durante todo esse período, duras foram as críticas quanto à limitação do método para revascularização das artérias coronárias sem circulação extracorpórea, no que diz, principalmente, à estabilização hemodinâmica (8-13).

Não existe, na literatura médica, uma explicação para o fato de o coração encontrar-se em posições totalmente anômalas e suportar essa situação por um longo período. A falta de dados para justificar esse fato deve-se ao motivo de ser uma técnica nova, a qual só agora começa a ser utilizada de maneira ampla em todo o mundo. Sendo assim, justifica-se o presente estudo, na proposta de apresentar respostas quanto às alterações hemodinâmicas sofridas pelo coração quando assume uma posição inteiramente atípica em relação à anatômica habitual.

O objetivo do presente trabalho é analisar o comportamento hemodinâmico intra-operatório do coração na revascularização do miocárdio sem a utilização da circulação extracorpórea.

\section{CASUÍSTICA E MÉTODOS}

No período de janeiro a março de 1999, foram operados, no Centro para Cirurgia Minimamente Invasiva, University at Buffalo, State University of
New York, 18 pacientes portadores de angina do peito e submetidos a revascularização miocárdica sem a utilização extracorpórea. Foi analisado o comportamento intra-operatório das pressões do coração. A idade variou de 40 a 81 anos, com média de 60,50 anos $\pm 14,70$ anos. Dez (55,55\%) pacientes eram do sexo feminino e $8(44,45 \%)$ do masculino. O peso variou de $68 \mathrm{~kg}$ a $147 \mathrm{~kg}$, com média de $98,83 \pm 21,9 \mathrm{~kg}$. A angina foi classificada segundo CCS, estando $1(5,56 \%)$ paciente na classe I, 1 $(5,56 \%)$ na classe II, $8(44,44 \%)$ na classe III e 8 $(44,44 \%)$ na classe IV. Na classificação da NYHA, $11(61,11 \%)$ pacientes estavam na classe I, 3 $(16,67 \%)$ na classe II, $2(11,11 \%)$ na classe III, 2 $(11,11 \%)$ na classe IV. A fração de ejeção variou de $20 \%$ a $68 \%$, com média de $44,22 \%$ e o desvio padrão de $14,70 \%$.

A doença era uniarterial em 1 (5,56\%) paciente, biarterial em $3(16,67 \%)$ e triarterial em 14 $(77,77 \%)$. Quarenta e nove artérias foram revascularizadas, com média de 2,72 pontes por paciente, sendo $15(30,61 \%)$ condutos arteriais (artéria torácica interna esquerda em 14 e artéria torácica interna direta em 1) e 34 enxertos venosos $(69,39 \%)$. A artéria interventricular anterior foi revascularizada em $18(100 \%)$ pacientes, a artéria circunflexa foi revascularizada em $13(72,22 \%)$, a coronária direita em $11(61,11 \%)$, sendo em 6 $(33,33 \%)$ pacientes o ramo interventricular posterior, e o ramo diagonal em 6 (38,88\%) doentes.

Foram identificados nos pacientes nos pacientes 10 fatores de risco cirúrgico: hipertensão arterial sistêmica em $12(66,66 \%)$, infarto do miocárdio prévio em $11(61,11 \%)$, obesidade em $8(44,44 \%)$, diabete melito em $7(38,88 \%)$, acidente vascular cerebral em $6(16,66 \%)$, doença vascular periférica em $3(16,66 \%)$, insuficiência mitral em 2 (11,11\%), alcoolismo em $2(11,11 \%)$, re-operação em $1(5,55 \%)$ e uso de balão intraaórtico em 1 (5,55\%).

\section{Avaliação Hemodinâmica Transoperatória}

A monitorização hemodinâmica foi realizada através da cateterização da artéria pulmonar com o cateter de Swan-Ganz. O cateter de três lúmens, com termistor incorporado, permitiu a avaliação dos seguintes parâmetros hemodinâmicos: freqüência cardíaca, débito cardíaco, pressão arterial média, pressão média de artéria pulmonar, pressão capilar pulmonar, pressão venosa central, resistência vascular sistêmica, resistência vascular pulmonar, "stroke volume" e índice cardíaco.

Estes parâmetros foram medidos em cinco diferentes momentos durante $o$ ato operatório: 
Lima R C, Escobar M A S, Della Santa R S, Diniz R, D’Aconda G, Bergsland J, Salerno T - Avaliação hemodinâmica intra-operatória na cirurgia de revascularização miocárdica sem auxílio da circulação extracorpórea. Rev Bras Cir Cardiovasc 2000; 15 (3): 201 -11.

1) Logo após a esternotomia, estando o coração na sua posição normal. Essa medição foi considerada de índice padrão e denominada de posição normal inicial (PNI);

2) Estando o coração na posição para revascularização da coronária interventricular anterior. Essa medição foi chamada de índice interventricular anterior (IVA);

3) Estando o coração na posição para revascularização da coronária direita. Essa medição foi chamada de índice coronária direita (CD);

4) Estando o coração na posição para revascularização da circunflexa e seus ramos. Essa medição foi chamada de índice circunflexa (Cx);

5) Após o término de todas as anastomoses segundo a técnica descrita, estando o coração de volta à sua posição normal. Essa medição foi chamada de índice posição final normal (PFN).

A medição dos parâmetros hemodinâmicos foi intermitente, pela técnica de termodiluição. O cateter utilizado em todos os pacientes foi da marca Baxter, "Thermodilution Paceport Catheters", modelo $93 \mathrm{~A}-931-7.5 \mathrm{~F}$ e $93 \mathrm{~A}-931 \mathrm{H}-7.5 \mathrm{~F}$.

Para realização das medições dos parâmetros, o balão foi insuflado com 1,5 cc para determinar uma boa curva de capilar pulmonar e em seguida injetados $20 \mathrm{cc}$ de solução salina fria através do cateter. Foram feitas as leituras no monitor e, para cada posição assumida pelo coração, foram feitas no mínimo três medições, assumindo-se a média destas, desprezando-se qualquer valor fora da média.

Todas as medições foram realizadas com aparelhos "Hewllet Packard" M1176A, Model 68 e Model M10994B.

\section{RESULTADOS}

\section{Monitorização Hemodinâmica}

\section{Freqüência Cardíaca}

A freqüência cardíaca manteve-se elevada durante todos os momentos de posicionamento do coração: posição normal inicial, posição de exposição do ramo interventricular anterior, posição de exposição da artéria coronária direita, posição de exposição da artéria circunflexa e na posição normal final. Essa elevação só apresentou significação estatística na posição de exposição da artéria coronária direita $(p=0,02)$ e na posição normal final $(p=0,0007)$ - (Gráfico 1).
GRÁFICO 1

AFERIÇÃO DA FREQÜÊNNCIA CARDÍACA (FC) DURANTE A REVASCULARIZACCÃO MIOCÁRDICA SEM CIRCULAÇÃO EXTRACORPÓREA. (PNI = POSIÇÃO NORMAL INICIAL, IVA = INTERVENTRICULAR ANTERIOR, CD = CORONÁRIA DIREITA, CX = CIRCUNFLEXA, PNF = POSIÇÃO NORMAL FINAL)

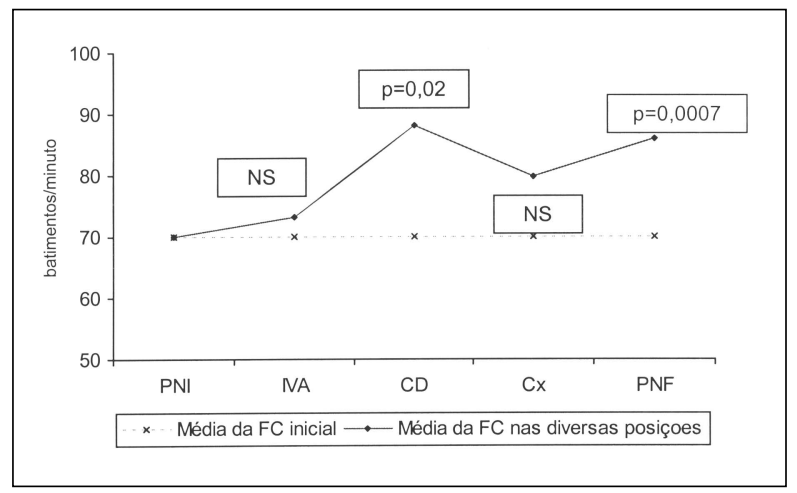

\section{Débito Cardíaco}

O débito cardíaco ao longo do procedimento apresentou variação mínima nos diversos momentos de posicionamento do coração e exposição das artérias coronárias: interventricular anterior, coronária direita e circunflexa. Entretanto, com o coração na posição normal final, observou-se um aumento importante do débito cardíaco com $(p=0,010)$ - (Gráfico 2).

\section{GRÁFICO 2}

MEDIÇÃO DO DÉBITO CARDÍACO (DC) DURANTE A REVASCULARIZAÇÃO MIOCÁRDICA SEM CIRCULAÇÃO EXTRACORPÓREA (PNI = POSIÇÃO NORMAL INICIAL, IVA = INTERVENTRICULAR ANTERIOR,

$C D=$ CORONÁRIA DIREITA, CX = CIRCUNFLEXA $P N F=P O S I C ̧ A \tilde{O}$ NORMAL FINAL)

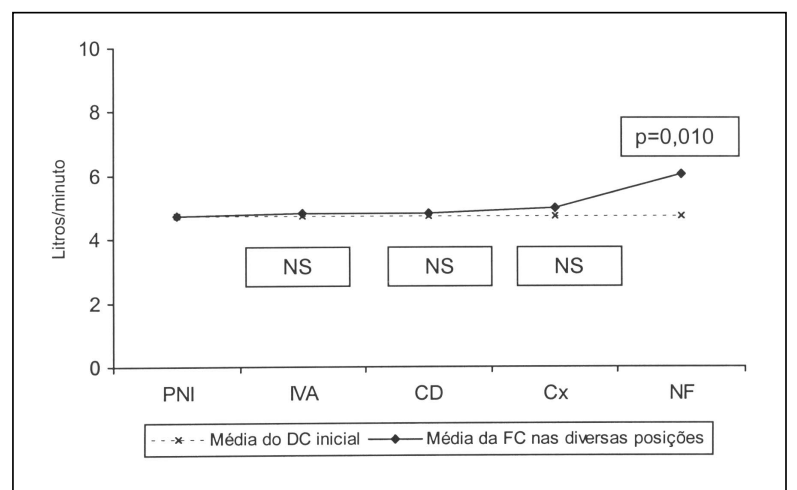

\section{Pressão Arterial Média}

A pressão arterial média (PAM) apresentou-se diminuída em todos os momentos do procedimento de exposição das artérias coronárias: interventricular anterior, coronária direita, artéria circunflexa e na posição 
Lima R C, Escobar M A S, Della Santa R S, Diniz R, D’Aconda G, Bergsland J, Salerno T - Avaliação hemodinâmica intra-operatória na cirurgia de revascularização miocárdica sem auxílio da circulação extracorpórea. Rev Bras Cir Cardiovasc 2000; 15 (3): 201 -11.

normal final. Essa diminuição apresentou significação estatística no momento da realização da anastomose da artéria interventricular anterior $(p=0,022)$ e na posição normal final $(p=0,040)$ - (Gráfico 3).

\section{GRÁFICO 3}

AFERICÃO DA PRESSÃO ARTERIAL MÉDIA (PAM)

DURANTE A REVASCULARIZAÇÃO MIOCÁRDICA SEM

CIRCULACÃO EXTRACORPOREA (PNI = POSIÇ̃̃O

NORMAL INICIAL, IVA = INTERVENTRICULAR ANTERI-

OR, CD = CORONÁRIA DIREITA, CX = CIRCUNFLEXA,

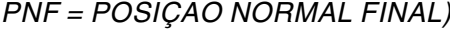

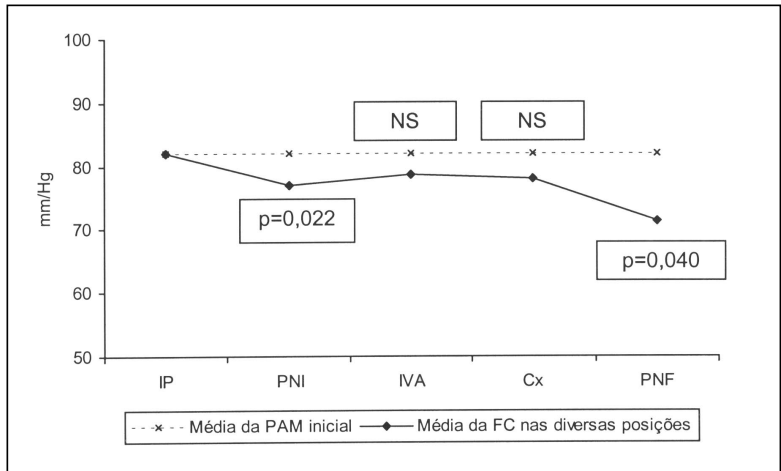

\section{Pressão Arterial Pulmonar Média}

A pressão arterial pulmonar (PAP) apresentouse diminuída durante todos os momentos de mobilização do coração, com a finalidade de exposição das artérias: interventricular anterior, coronária direita, artéria circunflexa. Durante essa mobilização, as diferenças não apresentaram significância estatística. O valor da PAP retornou praticamente ao mesmo valor da posição inicial, quando o coração retornou à sua posição normal (Gráfico 4).

\section{GRÁFICO 4}

AFERICÃO PRESSÃO ARTERIAL PULMONAR (PAP) DURANTE A REVASCULARIZACÃO MIOCÁRDICA SEM CIRCULACẼO EXTRACORPOREA (PNI = POSIÇÃO NORMAL INICIAL, IVA = INTERVENTRICULAR ANTERIOR, $C D=$ CORONÁRIA DIREITA, CX = CIRCUNFLEXA, $P N F=P O S I C ̧ A \tilde{O}$ NORMAL FINAL)

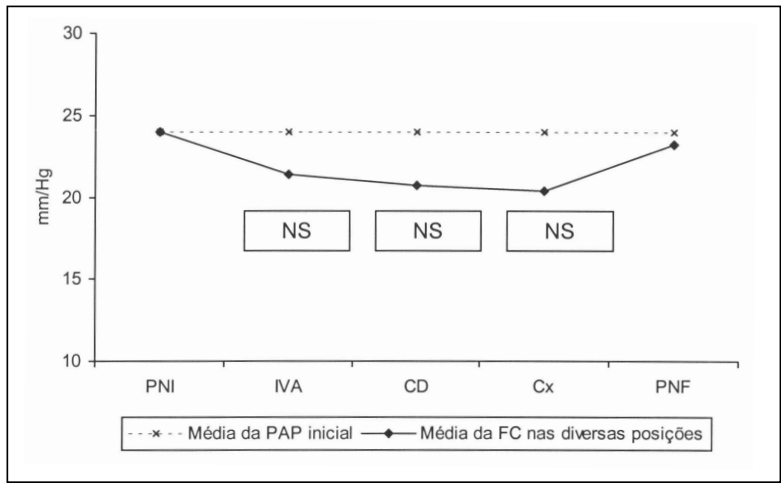

\section{Pressão Capilar Pulmonar Média}

A pressão capilar pulmonar (PCP) oscilou bastante durante a exposição das coronárias: interventricular anterior, coronária direita, artéria circunflexa e na posição normal final. Observou-se um aumento da PCP durante a realização das anastomoses da interventricular anterior e da coronária circunflexa. Durante a realização da anastomose da coronária direita e no retorno do coração à sua posição normal, foi observada uma diminuição da PCP. As diferenças não foram significativas estatisticamente (Gráfico 5).

\section{GRÁFICO 5}

MEDICÃO DA PRESSÃO CAPILAR PULMONAR (PCP) DURANTE A REVASCULARIZAĈ̃O MIOCÁRDICA SEM CIRCULAÇÃO EXTRACORPÓREA (PNI = POSIÇÃO NORMAL INICIAL, IVA = INTERVENTRICULAR ANTERIOR, CD = CORONÁRIA DIREITA, CX - CIRCUNFLEXA, $P N F=P O S I C C A O O N O R M A L$ FINAL)

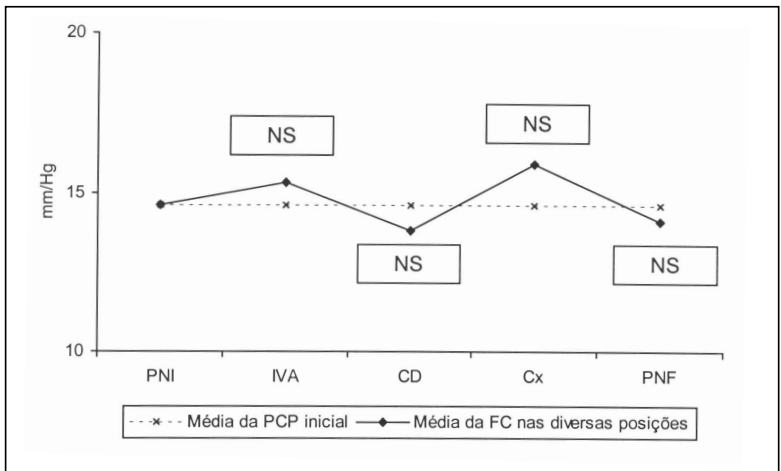

\section{Pressão Venosa Central}

A pressão venosa central (PVC) comportou-se de maneira mais variada durante a exposição das artérias: interventricular anterior, coronária direita, artéria circunflexa e na posição normal final. Apresentou-se elevada durante a revascularização da artéria circunflexa e seus ramos, diminuída durante a revascularização da interventricular anterior e inalterada durante a revascularização da coronária direita. Quando o coração retornou à sua posição original, observou-se queda da PVC. Não foi observada nenhuma diferença estatisticamente significativa quando se comparou os diversos momentos (Gráfico 6).

\section{Resistência Vascular Sistêmica}

A resistência vascular sistêmica (RVS) apresentou-se diminuída durante todo o procedimento, quando se mobilizou o coração para exposição das artérias: interventricular anterior, coronária direita, 
Lima R C, Escobar M A S, Della Santa R S, Diniz R, D’Aconda G, Bergsland J, Salerno T - Avaliação hemodinâmica intra-operatória na cirurgia de revascularização miocárdica sem auxílio da circulação extracorpórea. Rev Bras Cir Cardiovasc 2000; 15 (3): 201 -11.

\section{GRÁFICO 6}

AFERIÇÃO DA PRESSÃO VENOSA CENTRAL (PVC) DURANTE A REVASCULARIZACÃO MIOCÁRDICA SEM CIRCULAÇÃO EXTRACORPÓREA (PNI = POSIÇÃO NORMAL INICIAL, IVA = INTERVENTRICULAR ANTERIOR, CD = CORONÁRIA DIREITA, CX=CIRCUNFLEXA, $P N F=P O S I C ̧ A \tilde{O}$ NORMAL FINAL)

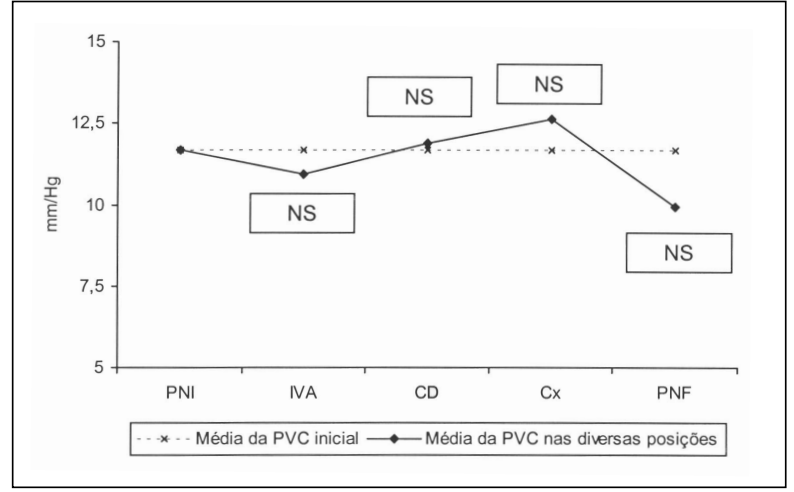

artéria circunflexa e na posição normal final. Essa diminuição foi estatisticamente significativa quando se realizou as anastomoses da coronária direita $(p=0,041)$, artéria circunflexa $(p=0,006)$ e quando o coração retornou a sua posição final $(p=0,0001)$ (Gráfico 7).

\section{GRÁFICO 7}

AVALIAÇÃO DA RESISTÊNCIA VASCULAR SISTÊMICA (RVS) DURANTE A REVASCULARIZACÃO, IVA = INTERVENTRICULAR ANTERIOR, CD = CORONÁRIA DIREITA, CX = CIRCUNFLEXA, PNF = POSIÇÃO NORMAL)

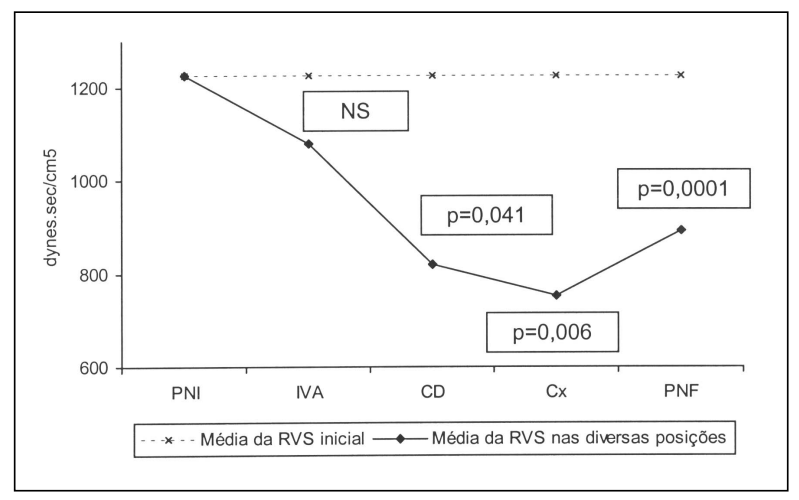

\section{Resistência Vascular Pulmonar}

A resistência vascular pulmonar (RVP) apresentou-se diminuída em todos os momentos do procedimento quando se mobilizou o coração para exposição das artérias: interventricular anterior, coronária direita, artéria circunflexa e na posição normal final. Entretanto, só apresentou significação estatística durante a realização da anastomose da interventricular anterior $(p=0,033)$ e quando o coração retornou à sua posição final $(p=0,002)$ - (Gráfico 8$)$.

\section{GRÁFICO 8}

MEDIÇÃO DA RESISTÊNCIA VASCULAR PULMONAR (RVP) NA REVASCULARIZACÃO MIOCÁRDICA SEM CIRCULAÇÃO EXTRACORPÓREA (PNI = POSIÇÃO NORMAL INICIAL, IVA = INTERVENTRICULAR ANTERIOR, CD = CORONÁRIA DIREITA, CX = CIRCUNFLEXA, $P N F=P O S I C ̧ A \tilde{O}$ NORMAL FINAL)

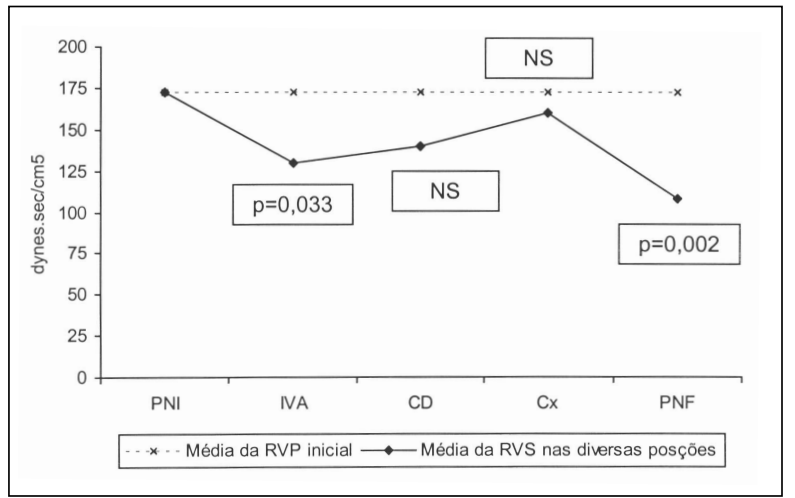

\section{"Stroke Volume"}

A "stroke volume" (SV) apresentou-se inalterado durante a anastomose da interventricular anterior. Durante a mobilização do coração para exposição das artérias: coronária direita, artéria circunflexa e na posição normal final, observou-se uma diminuição do "stroke volume" com tendência a normalidade na posição normal final. Só foram observadas diferenças estatísticas na coronária direita $(p=0,002)$ e artéria circunflexa $(p=0,0006)$ e seus ramos (Gráfico 9$)$.

\section{GRÁFICO 9}

MEDIÇÃO DO "STROKE VOLUME" (SV) DURANTE A REVASCULARIZACÃO MIOCÁRDICA SEM CIRCULAĈ̃O EXTRACORPÓREA (PNI = POSIÇÃO NORMAL INICIAL, IVA = INTERVENTRICULAR ANTERIOR, CD = CORONÁRIA DIREITA, CX CIRCUNFLEXA, PNF = POSIÇÃO NORMAL FINAL)

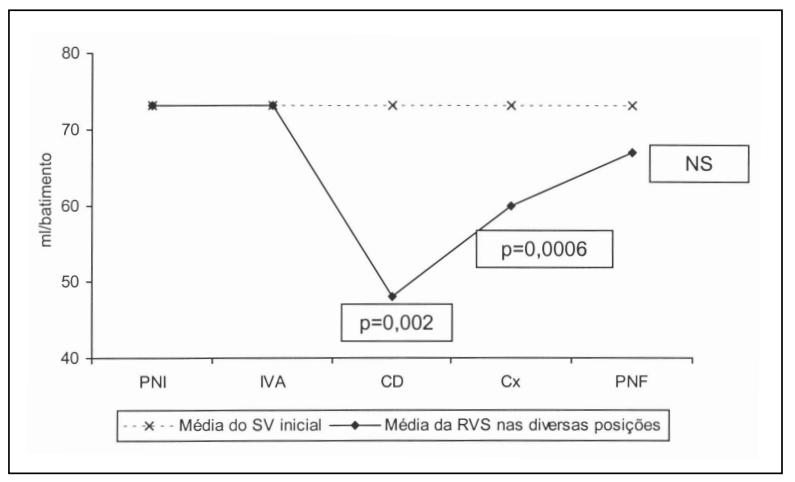


Lima R C, Escobar M A S, Della Santa R S, Diniz R, D’Aconda G, Bergsland J, Salerno T - Avaliação hemodinâmica intra-operatória na cirurgia de revascularização miocárdica sem auxílio da circulação extracorpórea. Rev Bras Cir Cardiovasc 2000; 15 (3): 201 -11.

\section{Índice Cardíaco}

O índice cardíaco (IC) apresentou-se diminuído durante o procedimento, quando se mobilizou o coração para exposição das artérias: interventricular anterior, coronária direita e artéria circunflexa. $\mathrm{Na}$ posição normal final, o IC aumentou. As diferenças foram estatisticamente significativas durante a realização das anastomoses da coronária direita $(p=0,0011)$, artéria circunflexa com seus ramos $(p=0,037)$ e quando o coração retornou à sua posição normal final $(p=0,011)$ - (Gráfico 10).

\section{GRÁFICO 10}

AVALIAÇÃO DO ÍNDICE CARDÍACO (IC) NA REVASCULARIZACÃO MIOCÁRDICA SEM CIRCULACÃO EXTRACORPÓREA (PNI = POSIÇ̃̃O NORMAL INICIAL, IVA = INTERVENTRICULAR ANTERIOR, CD = CORONÁRIA DIREITA, PNF = POSIÇÃO NORMAL FINAL)

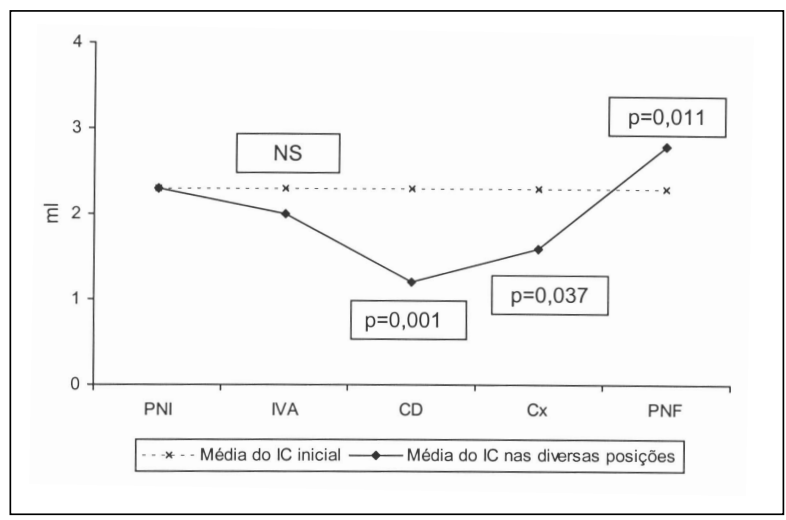

\section{COMENTÁRIOS}

A revascularização miocárdica sem CEC representa uma mudança de paradigma no tratamento da insuficiência coronária e nos últimos três anos, em todo o mundo, um grande interesse na utilização dessa técnica promoveu um importante desenvolvimento e aumento da aplicabilidade do método.

Por se tratar de uma operação onde é necessário se manusear o coração com maior intensidade e, estando a circulação sem o auxílio de algum tipo de suporte mecânico, é necessário se ter conhecimento das alterações hemodinâmicas ocorridas durante todo esse procedimento. No presente estudo, dez parâmetros hemodinâmicos foram analisados, os quais foram medidos em cinco diferentes momentos, sendo a primeira medição feita logo após a esternotomia com o coração no saco pericárdico (estando o pericárdio ainda fechado) na sua posição habitual e essa medição passou a ser considerada como o padrão porque o coração encontrava-se em condições próximas da normalidade. Em seguida, as medições foram feitas quando se posicionou o coração nas três posições básicas para realização das anastomoses (posição para realização da anastomose da interventricular anterior, da coronária direita e da artéria circunflexa e seus ramos). Por fim, foi feita a última medição com o coração de volta à sua posição normal no saco pericárdico. Obtidas essas medições, elas foram comparadas com a inicial que serviu de referência de padrão de normalidade. Com esse modelo de pesquisa, acreditou-se poder definir algumas modificações hemodinâmicas ocorridas durante a realização das anastomoses.

Foi utilizado, no presente estudo, o cateter de Swan-Ganz (14) com medição intermitente dos parâmetros hemodinâmicos, por se tratar de um método simples e de fácil manuseio. O cateter de Swan-Ganz permite avaliação de vários parâmetros, alguns diretos e outros indiretos. Foram medidos todos os parâmetros, embora neste estudo só tenham sido utilizados os seguintes: freqüência cardíaca, débito cardíaco, pressão arterial média, pressão média de artéria pulmonar, pressão capilar pulmonar, pressão venosa central, resistência vascular periférica, resistência vascular pulmonar, "stroke volume" e índice cardíaco. Não utilizamos as pressões arterial sistólica e diastólica, uma vez que na prática clinica em nosso país é utilizada a pressão arterial média. Seguindo essa mesma linha de definição, desprezamos a pressão atrial direita e valorizamos a pressão venosa central. $O$ débito cardíaco foi obtido de maneira muito simples em todos os pacientes e com relativa precisão. Foram tomadas todas as precauções, tomando-se pelo menos três medições e solicitando ao aparelho que fornecesse a média das medidas; dessa forma, procurouse eliminar qualquer erro de aferição.

Nos pacientes estudados a pressão arterial média apresentou-se diminuída durante as três posições básicas para realização das anastomoses e também quando o coração retornou à sua posição normal dentro do saco pericárdico. Os valores só tiveram significação estatística durante a posição para realização da anastomose da interventricular e na posição normal ao final do procedimento. É sabido que durante uma cirurgia pode ocorrer variação da pressão arterial devido a alterações do sistema nervoso central, cardíacas e respiratórias ${ }^{(8)}$. A anestesia contribui para essas alterações, principalmente pelo uso de drogas anestésicas e vasodilatadoras coronarianas e periféricas. No grupo em estudo observou-se acentuada queda na resistência vascular periférica durante todos os momentos da operação, o que justifica também uma diminuição da pressão arterial média durante todas as medições. Nesse caso, pode-se atribuir a diminuição da pressão devido a 
Lima R C, Escobar M A S, Della Santa R S, Diniz R, D’Aconda G, Bergsland J, Salerno T - Avaliação hemodinâmica intra-operatória na cirurgia de revascularização miocárdica sem auxílio da circulação extracorpórea. Rev Bras Cir Cardiovasc 2000; 15 (3): 201 -11.

vasodilatação periférica e provavelmente o manuseio do coração para realização das anastomoses coronárias sem CEC não tenha interferido de maneira significativa.

A freqüência cardíaca é uma das maneiras mais simples e eficazes de aumentar o débito cardíaco. Esse é, provavelmente, um mecanismo importante e o mais freqüentemente empregado para efetuar rápidas alterações no débito cardíaco ${ }^{(15)}$. Um aumento da freqüência cardíaca por si só é capaz de elevar em até três vezes o débito cardíaco; entretanto, acima de um determinado ritmo o débito pode começar a diminuir com maiores aumentos da freqüência. A diminuição do débito acima de um determinado ritmo é devida ao encurtamento da diástole, que limita o tempo para o enchimento adequado dos ventrículos, como também para a perfusão coronária que ocorre predominantemente na diástole, em particular no ventrículo esquerdo. $\mathrm{O}$ aumento do ritmo cardíaco pode também aumentar a contratilidade miocárdica, melhorando a performance ventricular e esse efeito é chamado de auto-regulação homeométrica. Nos pacientes em estudo observou-se um aumento da freqüência cardíaca em todos os momentos. A freqüência aumentou progressivamente, tendo variado entre 70 a 90 batimentos por minuto, o que, nos parece, os valores esperados para realização do procedimento. Ao contrário do passado, onde procurava-se operar com freqüência baixa fazendo-se uso de bloqueadores de cálcio, hoje, com o uso de estabilizadores cardíacos, pode-se operar esses pacientes com freqüência mais altas e possibilitar melhor performance cardíaca. A freqüência ideal para realização dessas anastomoses deve variar entre 60 e 90 batimentos por minuto. $\mathrm{O}$ aumento da freqüência durante o procedimento parece ter sido devido ao manuseio do paciente em geral, e não pela técnica de posicionamento do órgão, uma vez que a anestesia é conduzida de forma mais superficial, visando extubar esses doentes na sala de operações ou nas primeiras horas de pós-operatório.

A pressão arterial pulmonar pode ser influenciada por vários fatores, os quais podem também influenciar na resistência vascular pulmonar: os cardiocirculatórios, os da circulação brônquica colateral, os respiratórios, os vasomotores (extra e intrapulmonares) $(16,17)$. Nos casos estudados dois fatores devem ter influenciado sobremaneira na queda da pressão arterial pulmonar: os cardiocirculatórios e os vasomotores. Pelos mesmos motivos relacionados a pressão arterial média, a anestesia fez uso de drogas vasodilatoras potentes, que possibilitaram a diminuição da resistência periférica, da pressão arterial média e da pressão do átrio esquerdo. Essas drogas tam- bém podem atuar no sistema pulmonar, diminuindo a resistência vascular pulmonar. Portanto, a queda da pressão arterial pulmonar média, em comparação com o valor medido inicialmente e reconhecido como padrão nos pacientes estudados, deve estar relacionada com fatores extrínsecos ao procedimento de revascularização miocárdica sem CEC. Essas variações não apresentaram significância estatística e a pressão arterial pulmonar média tendeu a se normalizar logo após o coração retornar à sua posição normal no saco perícárdico.

A resistência vascular pulmonar encontrouse diminuída em todas as fases da observação nos pacientes operados. Em dois momentos a diminuição foi mais acentuada e apresentou significação estatística: na realização da anastomose do ramo interventricular anterior e na posição final. Não foi observada nenhuma explicação fisiológica para tal, a não ser resultado do efeito das drogas utilizadas pela anestesia, que proporcionaram uma acentuada vasodilatação da vasculatura periférica e pulmonar.

Foi observada elevação do débito cardíaco quando o coração retornou à sua posição normal $e$ os valores foram significativos do ponto de vista hemodinâmico. Não se tem uma explicação fisiológica para o aumento do débito após o retorno do coração ao saco pericárdico ${ }^{(18)}$, mas, no momento, pode-se afirmar que a manipulação do coração para a realização das diversas anastomoses não alterou o débito cardíaco, mesmo com a exposição da artéria circunflexa, onde se tem a expressão máxima no manuseio cardíaco na técnica utilizada.

A curva da função cardíaca é definida pelo débito cardíaco e a pressão de átrio direito ou pressão venosa central (pré-carga). Esse conjunto é a própria expressão da curva de FrankStarling (19), a qual é uma característica do próprio coração e pode ser estudada isoladamente do resto da circulação. O "stroke volume" não apresentou nenhuma alteração durante a revascularização do ramo interventricular anterior, mas, durante a anastomose da artéria coronária direita, observou-se uma acentuada redução com significação estatística, porém sem nenhuma expressão clínica. Essa curva tendeu a normalizar durante 0 momento da circunflexa e na posição final, embora ainda tenha sido significante durante a anastomose da circunflexa e seus ramos. Isso significa que a posição da artéria coronária direita e da circunflexa alterou a quantidade de sangue bombeado pelo coração em cada sístole; entretanto, o débito cardíaco não foi alterado porque houve um aumento da freqüência cardíaca. Portanto, é importante se ter em mente que atualmente se deve operar esses pacientes com fre- 
Lima R C, Escobar M A S, Della Santa R S, Diniz R, D’Aconda G, Bergsland J, Salerno T - Avaliação hemodinâmica intra-operatória na cirurgia de revascularização miocárdica sem auxílio da circulação extracorpórea. Rev Bras Cir Cardiovasc 2000; 15 (3): 201 -11.

qüências mais altas e isso é possível graças ao uso dos estabilizadores cardíacos. O índice cardíaco também apresentou uma curva com comportamento muito similar ao "stroke volume"; no entanto, após a realização da anastomose da artéria coronária direita, esse índice tendeu a se normalizar, finalizando com valores maiores do que o valor inicial padrão. Essas diferenças foram estatisticamente significativas nos três momentos finais.

As mudanças ocorridas na resistência periférica provocam alterações na curva de função vascular e essas alterações são complexas porque as funções cardíaca e vascular se alteram. As arteríolas possuem somente $3 \%$ do volume total de sangue do corpo. Para uma mesma pressão venosa central, um aumento da resistência periférica (vasoconstrição) determina diminuição do débito cardíaco e, da mesma forma, diminuição da resistência periférica (vasodilatação), permitindo aumento do débito cardíaco. A resistência vascular sistêmica apresentou acentuada diminuição em todos os momentos do procedimento, quando se comparou ao índice padrão, chegando a uma redução de quase $40 \%$. Durante a realização das anastomose da artéria coronária direita, artéria circunflexa e seus ramos, foram os momentos de maior redução e foram significativos do ponto de vista estatístico. Chama a atenção que o coração, quando retornou à posição normal, houve tendência da resistência vascular periférica aumentar, porém os valores ainda eram muito reduzidos e apresentaram significação estatística.

Existe uma grande relação entre o débito cardíaco e o retorno venoso e geralmente a quantidade de sangue bombeado pelo ventrículo esquerdo é semelhante à aquela que aporta ao coração direito. Sendo a circulação um sistema fechado, o retorno venoso ao coração tem que ser igual ao débito cardíaco num mesmo intervalo de tempo. Por sua vez, o fluxo total de sangue em todo o sistema depende da capacidade de bombeamento do coração, das características do circuito e do volume total de fluido do sistema. Sendo assim, débito cardíaco e retorno venoso são dois simples elementos analisados dentro de um sistema complexo. Sendo o débito cardíaco o volume de sangue impulsionado pelo coração por unidade tempo e o retorno venoso a quantidade de sangue que retorna ao coração em unidade de tempo, o equilíbrio só é conseguido quando esses dois fluxos são iguais. Mudanças agudas na contratilidade miocárdica, na resistência periférica ou no volume de sangue podem afetar transitoriamente o débito cardíaco e o retorno venoso. A pressão venosa central apresentou um comporta- mento da maneira mais variada. Durante a realização da anastomose da interventricular anterior, ela apresentou-se diminuída. Durante a anastomose da artéria coronária direita, os valores foram semelhantes ao valor inicial com o pericárdio fechado, demonstrando que o posicionamento e a tração realizada na borda do ventrículo direito não interferiram na pré-carga e, conseqüentemente, no enchimento de átrio e ventrículo direito. No momento de realização da anastomose da artéria circunflexa e seus ramos, a pressão venosa central apresentou-se um pouco elevada em relação ao índice padrão, vindo a cair drasticamente ao final do procedimento, quando o coração foi colocado na sua posição normal. Toda essas alterações para mais e para menos não foram significativas. A pressão capilar pulmonar nos pacientes operados também teve comportamento oscilatório semelhante. Durante a realização das anastomoses da interventricular anterior e artéria circunflexa, a pressão capilar pulmonar apresentou pequeno aumento e, durante a realização da anastomose da artéria coronária direita e na posição final, se observou uma pequena diminuição. Essas variações não tiveram repercussão clínica e também não apresentaram significação estatística. Vale salientar que, mesmo durante a realização das anastomose da parede posterior do coração, onde este encontra-se inteiramente fora do tórax, em posição de "ectopia cordis", o ventrículo esquerdo não apresentou nenhuma disfunção, significando que a operação pode ser realizada com segurança.

Qualquer mudança na contratilidade afeta os dois ventrículos de forma que irá provocar uma distribuição diferente do volume de sangue dentro dos dois sistemas vasculares. Por exemplo, no infarto agudo do miocárdio a contratilidade do ventrículo esquerdo diminuirá e será instalada uma falência ventricular esquerda. De imediato a pressão do átrio esquerdo não muda, mas o ventrículo esquerdo começará a bombear menos sangue enquanto o ventrículo direito continuará enviando a quantidade de sangue normal. Nesse momento, a pressão de átrio esquerdo se elevará e a de átrio direito diminuirá. Em um momento seguinte, a elevação da pressão de átrio esquerdo será acompanhada de elevação da pressão venosa pulmonar, a qual pode causar sérias conseqüências clinicas. $\mathrm{Na}$ insuficiência cardíaca, o coração não se encontra em condições de prover adequadamente a quantidade de sangue para os tecidos e pode ocorrer de forma aguda e crônica. Na oclusão coronária aguda, o volume de sangue não muda imediatamente, mas a análise das funções cardíaca e vascular denotará diminuição do débito cardíaco. $\mathrm{Na}$ insuficiência cardíaca crônica, também acontece mudança nas funções cardíaca e vascular; entretanto, apesar da curva 
Lima R C, Escobar M A S, Della Santa R S, Diniz R, D’Aconda G, Bergsland J, Salerno T - Avaliação hemodinâmica intra-operatória na cirurgia de revascularização miocárdica sem auxílio da circulação extracorpórea. Rev Bras Cir Cardiovasc 2000; 15 (3): 201 -11.

mudar o débito cardíaco, pode se encontrar mais elevado do que na insuficiência aguda.

Não é correto analisar-se o débito cardíaco isoladamente; deve-se levar em conta as alterações da freqüência cardíaca, uma vez que esta, por si só, é capaz de interferir em outros três fatores: pré-carga, pós-carga e contratilidade. Esses fatores determinam o "stroke volume", o que faz com que seja complexa a análise de todos esses parâmetros. Por exemplo, o aumento da freqüência cardíaca poderá diminuir a duração da diástole, diminuindo o enchimento ventricular e, conseqüentemente, a pré-carga. Se a elevação da freqüência cardíaca provoca um aumento no débito cardíaco, a pressão arterial irá se alterar e também a póscarga. Finalmente, o aumento da freqüência cardíaca poderá aumentar o influxo de cálcio por minuto dentro da célula miocárdica e, por conseguinte, aumentar a contratilidade miocárdica. Por outro lado, o aumento da freqüência cardíaca a níveis exageradamente elevados pode diminuir o débito cardíaco (20).

\section{CONCLUSÕES}

Com base na experiência acumulada nesta série consecutiva de pacientes e nas observações hemodinâmicas feitas em 18 pacientes, podemos concluir que:

1. A técnica presente permite a mobilização máxima do coração sem indução de instabilidade hemodinâmica;

2. A melhora de alguns parâmetros hemodinâmicos ao final do procedimento, pode ser justificada:

- Devido a resposta à revascularização miocárdica;

Decorrente da liberação de catecolaminas após a manipulação do coração nas diversas posições;

- Decorrente da liberação de mediadores vasoativos depois da tração prolongada do pericárdio.

Lima R C, Escobar M A S, Della Santa R S, Diniz R, D’Aconda G, Bergsland J, Salerno T - Intraoperative hemodynamic evaluation of myocardial revascularization without extracorporeal circulation. Rev Bras Cir Cardiovasc 2000; 15 (3): 201-11

ABSTRACT: Background: To analyze the hemodynamic behavior of the heart in revascularization of the myocardium without use of extracorporeal circulation.

Material And Methods: From August 1991 to June 1999, 616 patients suffering from angina of the chest underwent revascularization of the myocardium without use of extracorporeal circulation. In 18 patients the intraoperative hemodynamic parameters were studied.

Results: Heart rate remained high at all times during positioning of the heart $(p=0.0007)$. Cardiac output throughout the procedure presented a very slight variation at different moments of positioning of the heart and exposure of the coronary arteries. However, once the heart was in its final normal position a major increase in output was noted $(p=0.010)$. Mean blood pressure was found to be decreased throughout the procedure for exposing the coronary arteries $(p=0.022)$. Arterial pulmonary pressure proved to be reduced at all times during mobilization, but without statistical significance. Pulmonary capillary pressure showed considerable, but statistically insignificant variation during exposure of the arteries. Central venous pressure behaved in a more varied fashion during exposure of the arteries, but again without statistical significance. Systemic vascular resistance proved to be diminished throughout the procedure $(p=0.0001)$. Pulmonary vascular resistance remained reduced at all times during the procedure $(p=0.002)$. Stroke volume was unchanged during anastomosis of the anterior interventricular artery and statistically significant differences were observed only in the right coronary $(p=0.002)$ and right circumflex $(p=0.0006)$ artery and its branches. The cardiac index was shown to be diminished throughout the procedure $(p=0.0011)$.

Conclusions: A) The present technique allows for maximum mobilization of the heart without inducing hemodynamic instability. B) The improvement of a number of hemodynamic parameters at the end of the procedure may be accounted for by: (1) the response to the myocardial revascularization; (2) the release of catecholamines following manipulation of the heart in the different positions and (3) the release of vasoactive mediators following prolonged traction of the pericardium.

DESCRIPTORS: Myocardial revascularization, methods. Heart, physiopathology. Extracorporeal circulation. Hemodynamics. Intraoperative period. 
Lima R C, Escobar M A S, Della Santa R S, Diniz R, D’Aconda G, Bergsland J, Salerno T - Avaliação hemodinâmica intra-operatória na cirurgia de revascularização miocárdica sem auxílio da circulação extracorpórea. Rev Bras Cir Cardiovasc 2000; 15 (3): 201 -11.

\section{REFERÊNCIAS BIBLIOGRÁFICAS}

1 Kolessov V I - Mammary artery-coronary artery anastomosis as method of treatment for angina pectoris. $J$ Thorac Cardiovasc Surg 1967; 54: 535-44.

2 Ankeney J L - Coronary vein graft without cardiopulmonary bypass. Surgical motion picture presented at Annual Meeting of the Society of Thoracic Surgeons. Ann Thorac Surg 1968; 5: 443-8.

3 Trapp W G \& Bisarya R - Placement of coronary artery bypass graft without pump oxigenator. Ann Thorac Surg 1975; 19: 1-9.

4 Buffolo E, Andrade J C, Succi J E et al. - Revascularização direta do miocárdio sem circulação extracorpórea: descrição da técnica e resultados iniciais. Arq Bras Cardiol 1982; 38: 365-73.

5 Benetti F J - Direct coronary surgery with saphenous vein bypass without either cardiopulmonary bypass or cardiac arrest. J Cardiovasc Surg 1985; 26: 217-22.

6 Lima R, Escobar M, Wanderley Neto $\mathrm{J}$ et al. Revascularização do miocárdio sem circulação extracorpórea. An Fac Med CCS UFPE 1993; 38: 325.

7 Lima R, Escobar M, Wanderley Neto J et al. Revascularização do miocárdio sem circulação extracorpórea: resultados imediatos. Rev Bras Cir Cardiovasc 1993; 8: 171-8.

8 Benetti F J, Ballester C, Sani G, Doonstra P, Grandjean $\mathrm{J}$ - Video assisted coronary bypass surgery. J Card Surg 1995; 10: 620-5.

9 Lima R, Granja L, Escobar M et al. - Revascularização da artéria circunflexa sem auxílio da circulação extracorpórea. In: Anais do XV Congresso da Sociedade Norte/Nordeste de Cardiologia, VII Congresso da Sociedade de Cardiologia do Estado da Bahia, VII Jornada N/NE de Enfermagem em Cardiologia, IV Jornada N/NE de Fisioterapia Cardiovascular, II Encontro N/NE de Terapia Intensiva em Cardiologia e Cirurgia Cardíaca. Salvador, Bahia, Julho de 1995. (Resumos).

10 Lima R - Revascularização da artéria circunflexa sem auxílio da CEC. In: Anais do XII Encontro dos Discípulos do Dr. E. J. Zerbini. Curitiba, Paraná: Sociedade dos Discípulos do Dr. E. J. Zerbini, 1995. p. 6. (Resumos).

11 Granja L, Lima R, Escobar M et al. - Revascularização do ramo marginal da artéria circunflexa sem auxílio da extracorpórea. (CEC): experiência inicial. In: Anais do $23^{\circ}$ Congresso Nacional de Cirurgia Cardíaca. Recife, Pernambuco: Sociedade Brasileira de Cirurgia Cardiovascular, 1996. p. 33-4. (Resumos).

12 Lobo Filho J G, Dantas M C B R, Rolim J G V et al. Cirurgia de revascularização completa do miocárdio sem circulação extracorpórea: uma realidade. Rev Bras Cir Cardiovasc 1997; 12: 15-21.
13 Lima R C - Padronização técnica de revascularização miocárdica da artéria circunflexa e seus ramos sem circulação extracorpórea. [Tese. Doutorado]. São Paulo: Escola Paulista de Medicina da Universidade Federal de São Paulo,1999.

14 Swan H J - Baloon flotation catheters: their use in hemodynamic monitoring in clinical practice. JAMA 1975; 233: 856-67.

15 Terzi R \& Araújo S - Monitorização hemodinâmica e suporte cardiocirculatório do paciente crítico. São Paulo: Editora Ateneu, 1996. 360p.

16 Hussay B A, Barcia R C, Covian M R et al. - Fisiologia Humana. 4. ed. Buenos Aires: El Ateneo, 1971. $1318 p$.

17 Hurst J W, Logue R B, Schlant R C, Wenger N K, eds. O coração e as veias. 3. ed. Mcgraw Hill, Inc: 1974. p.920.

18 Guyton A C, Jones C E, Coleman T G, eds. Circulatory physiology: cardiac output and its regulation. 2. ed. Philadelphia: Saunders, 1971. v.4.

19 Kaye W - Invasive monitoring techniques: arterial cannulation, bedside pulmonary artery catheterization, and arterial puncture. Heart Lung 1983; 12: 395-427.

20 Lozman J, Powers S R Jr., Older T et al. - Correlation of pulmonary wedge and left atrial pressures: a study in the patient receiving positive end expiratory pressure ventilation. Arch Surg 1974; 109: 270-7.

\section{Discussão (Transcrições de fita gravada)}

\section{DR. LIBERATO SAVIO SIQUEIRA DE SOUZA \\ Divinópolis, $M G$}

Vou realizar os comentários baseado nos dados clínicos propriamente ditos. Gostaria de saber se estes dados de final de operação permaneceriam inalterados na evolução pós-operatória imediata do paciente na UTI. Isto porque Calafiore descreve, em seu material, maior incidência de uso de aminas por pressão arterial baixa nos pacientes operados sem CEC, comparativamente aos pacientes com CEC. Também observamos este fato em nossa experiência de mais de 200 casos de operações realizadas sem CEC. Observamos, ainda, maior incidência de pacientes confusos ou agitados, principalmente em idosos submetidos a esta operação. Problemas de hipoxemia nas operações sem CEC também são descritos. Será que as trações que sofre o coração e a isquemia não seriam os responsáveis por isto? Quais dados de alteração hemodinâmica dariam indicação de que isto poderia ocorrer no futuro? Para quem realiza também a operação com CEC, um trabalho que também poderá ser realizado é a avaliação hemodinâmica com- 
Lima R C, Escobar M A S, Della Santa R S, Diniz R, D’Aconda G, Bergsland J, Salerno T - Avaliação hemodinâmica intra-operatória na cirurgia de revascularização miocárdica sem auxílio da circulação extracorpórea. Rev Bras Cir Cardiovasc 2000; 15 (3): 201 -11.

parativa entre os pacientes operados sem CEC, nas diversas fases apresentadas no trabalho do Dr. Ricardo, e nos pacientes operados com CEC, antes e após a interrupção desta. Por fim, gostaria de saber se o Dr. Ricardo realizou as medidas destes parâmetros em pacientes instáveis hemodinamicamente, como, por exemplo, coração muito irritável, grandes cardiomegalias, pacientes que já entram com baixo débito no ato cirúrgico e no edema agudo; caso tenha feito, o que foi observado?

\section{DR. TOMAS SALERNO \\ New York, USA}

Um dos maiores problemas da operação de coronária sem CEC era posicionar o coração, pela dificuldade de abordar a artéria circunflexa de maneira segura. Esta idéia do Dr. Ricardo Lima surgiu e mudou o conceito de como se faz a operação sem CEC. O trabalho do Dr. Ricardo é o primeiro, no mundo, que fala sobre a hemodinâmica dessa posição do coração. Ontem, na sessão do Dr. Jatene, houve uma grande discussão sobre uma hipotensão transiente - que é recuperável - quando se faz a posição com a técnica do Ricardo Lima. Este trabaIho mostrou, conclusivamente, que se pode fazer esta operação sem problemas hemodinâmicos, o que é confirmado dia a dia em nosso Serviço. Na minha opinião, esta é uma das maiores contribuições do Brasil, além do Dr. Enio Buffolo, em cirurgia sem CEC.

\section{DR. LIMA}

(Encerrando)

Não foi feita a análise pós-operatória destes doentes porque nos detemos unicamente a parte intra-operatória. Isto seria realmente um dado importante, mas não foi o objetivo, naquele momento, de nosso trabalho. Na verdade, estávamos curiosos em saber o que acontecia com as pressões do coração quando o coração era movido em diversas posições. Quando cheguei ao Brompton Hospital, como jovem cirurgião, tinha curiosidade para colocar o dedo para tocar o coração e o Christopher Lincoln batia o porta-agulhas no meu peito e dizia: "Don't touch the heart" porque ele pode fibrilar. Esta é uma coisa que todos nós aprendemos, nós que fazemos a operação sem CEC, manuseamos o coração extensivamente e o coração agüenta. Então, o que acontece intra-operatoriamente? Esta era a grande curiosidade e, por isso, nos detivemos unicamente nesses dados, mas acho que muita coisa pode ser feita nessa área; por exemplo, a medição contínua do débito, que, neste trabalho, foi seqüencial; a avaliação no pós-operatório também está aberta para estudos ulteriores. Com relação à agitação pós-operatória, na minha experiência com operação sem CEC de aproximadamente 700 pacientes, isto ocorreu em alguns doentes. Acho que nestes doentes talvez nós tenhamos exagerados um pouco na hipotensão, acreditando no velho paradigma da própria $\mathrm{CEC}$, que a freqüência e a pressão arterial deveriam ser baixadas. Hoje, eu acho que a pressão arterial tem que ficar realmente alta, para que haja perfusão cerebral. Um colega, que trabalha na Universidade de Tampa, opera todos os pacientes sem CEC, analisando o consumo de oxigênio pelo cérebro, usando aparelhos muito simples, que demonstram isto muito bem. Acredito que este é um dado muito importante, não se deve deixar a pressão cair. Esta foi uma série seguida de doentes, não houve nenhuma seleção, doente melhor ou pior; nesse grupo havia pacientes muito graves, obesos (o peso médio chegava quase a $90 \mathrm{~kg}$ ), e doentes em programa de hemodiálise. Havia doentes instáveis nesse grupo, mas não fiz essa diferenciação. O Dr. Penna perguntou sobre o uso de drogas vasoativas; se o doente estiver bem no plano anestésico, com freqüência de 80 , nosso conceito é de que não se deve baixar a freqüência nem a pressão. A freqüência elevada e a pressão não muito alta, entre 80 e $75 \mathrm{mmHg}$ de média, como nós usamos no Brasil, eu acho que é ideal. Caso o doente não esteja assim, estiver um pouco mais betabloqueado, entramos com dopamina $3 \mathrm{mcg} / \mathrm{kg}$ de peso para acelerar; a freqüência cardíaca se mantém elevada o tempo todo e, com isso, o débito tende a ficar estável durante toda a operação. Acho que este é um detalhe importante, pois evita os problemas cerebrais no pós-operatório, como o Dr. Liberato comentou. Todos os pacientes foram operados com uso de perfusores intracoronários; não posso determinar exatamente se teve alguma artéria na qual não se tenha conseguido colocar o perfusor, por ser uma artéria menor, mas em acima de $90 \%$ dos casos foram usados perfusores intracoronários. 